\title{
Lie algebras generated by Jordan operators
}

\author{
by \\ Peng CaO and Shanli Sun (Beijing)
}

\begin{abstract}
It is proved that if $J_{i}$ is a Jordan operator on a Hilbert space with the Jordan decomposition $J_{i}=N_{i}+Q_{i}$, where $N_{i}$ is normal and $Q_{i}$ is compact and quasinilpotent, $i=1,2$, and the Lie algebra generated by $J_{1}, J_{2}$ is an Engel Lie algebra, then the Banach algebra generated by $J_{1}, J_{2}$ is an Engel algebra. Some results for normal operators and Jordan operators on Banach spaces are given.
\end{abstract}

1. Introduction. Let $\mathcal{X}$ be a Banach space, and $\mathscr{H}$ be a Hilbert space. The Banach algebra of all bounded linear operators on $\mathcal{X}$ is denoted by $\mathbf{B}(\mathcal{X})$. It is a Lie algebra with the Lie product $\left[T_{1}, T_{2}\right]=T_{1} T_{2}-T_{2} T_{1}$ for $T_{1}, T_{2} \in \mathbf{B}(\mathcal{X})$. Let $M \subset \mathbf{B}(\mathcal{X})$. The Lie algebra generated by $M$, denoted by $\varepsilon(M)$, is the smallest Lie algebra containing $M$. Let $\mathcal{A}(M)$ denote the associative algebra generated by $M, \bar{M}$ the closure of $M$ in $\mathbf{B}(\mathcal{X})$, and $\operatorname{Ker}(T)$ the kernel of an operator $T$.

Recall that $T \in \mathbf{B}(\mathcal{X})$ is hermitian if $\|\exp (i t T)\|=1$ for every $t \in \mathbb{R}$, and hermitian-equivalent if $\sup _{t \in \mathbb{R}}\|\exp (i t T)\|<\infty$. An operator $N \in \mathbf{B}(\mathcal{X})$ is normal (resp., normal-equivalent) if $N=A+i B$, where $A, B$ are hermitian (resp., hermitian-equivalent), and $[A, B]=0$. Some basic properties of hermitian operators can be found in [8]; for hermitian-equivalent operators, see [4], [1].

We will also make use of the theory of decomposable operators. One can find the concepts of "spectral operator", "scalar operator", "generalized scalar operator", and "decomposable operator" in [6]. We will denote by $\mathcal{B}_{\text {ad } N}(F)$ the maximal spectral subspace of $\mathbf{B}(\mathcal{X})$ associated with ad $N$ and a closed subset $F \subset \mathbb{C}$. It is well known that if $N$ is a normal-equivalent operator, then so is ad $N$ by $[4, \S 14$, Proposition 4]. Then $N$ and ad $N$ are generalized scalar operators by $[4, \S 14$, Remark 6$]$. The following lemmas are useful.

2000 Mathematics Subject Classification: Primary 47B15; Secondary 47B47.

Key words and phrases: normal-equivalent operators, Engel Lie algebras, Jordan operators.

Project supported by National Natural Science Foundation of China No. 10571011 and No. 10601005. 
Lemma 1.1 ([4, $\S 14$, Theorem 6$])$. If $N \in \mathbf{B}(\mathcal{X})$ is normal-equivalent and $\sigma(N)=\{0\}$, then $N=0$.

Lemma 1.2 ([4, §14, Corollary 4$])$. If $N \in \mathbf{B}(\mathcal{X})$ is normal-equivalent, then

$$
\mathcal{B}_{N}(\{\lambda\})=\operatorname{Ker}(N-\lambda I)=\operatorname{Ker}(A-\operatorname{Re} \lambda)=\operatorname{Ker}(B-\operatorname{Im} \lambda)
$$

for each $\lambda$ in $\sigma(N)$.

If $N$ is normal-equivalent, then by [6, Lemma 4.4.4] we have

LEMMA 1.3.

$$
\mathcal{B}_{\text {ad } N}(\{0\})=\left\{S \in \mathbf{B}(\mathscr{H}) \mid \lim _{n \rightarrow \infty}\left\|(\operatorname{ad} N)^{n} S\right\|^{1 / n}=0\right\} .
$$

We can also define an involution on normal-equivalent operators in $\mathbf{B}(\mathcal{X})$, namely for a normal-equivalent operator $N \in \mathbf{B}(\mathcal{X})$ with $N=A+i B$, define $N^{*}=A-i B$.

Lemma 1.4 ([2, Theorem $])$. If $N_{1}, N_{2} \in \mathbf{B}(\mathcal{X})$ are normal-equivalent and $N_{1} S=S N_{2}$ for some $S \in \mathbf{B}(\mathcal{X})$, then $N_{1}^{*} S=S N_{2}^{*}$.

For a Lie algebra $\mathcal{L} \subset \mathbf{B}(\mathcal{X}), \overline{\mathcal{L}}$ is the closure of $\mathcal{L}$ in $\mathbf{B}(\mathcal{X})$. For every $T \in \mathcal{L}, \operatorname{ad} T: \mathcal{L} \rightarrow \mathcal{L}$ is quasinilpotent (i.e., $\left.\lim \left\|(\operatorname{ad} T)^{n}\right\|^{1 / n}=0\right)$ if and only if $\operatorname{ad} T: \overline{\mathcal{L}} \rightarrow \overline{\mathcal{L}}$ is quasinilpotent if and only if $\left\|(\operatorname{ad} T)^{n}(S)\right\|^{1 / n} \rightarrow 0$ as $n \rightarrow \infty$ for any $S \in \overline{\mathcal{L}}$. A Volterra operator is a compact quasinilpotent operator.

Recall that if $\mathcal{L}$ is a normed Lie algebra, then $\mathcal{L}$ is called ad-compact if the operator ad $a$ on $\mathcal{L}$ is compact for every $a \in \mathcal{L} ; \mathcal{L}$ is an Engel Lie algebra if ad $a$ is quasinilpotent for every $a \in \mathcal{L}$; and $\mathcal{L}$ is called E-solvable if every nonzero quotient of $\mathcal{L}$ by a closed ideal has a nonzero Engel ideal. Every finite-dimensional E-solvable Lie algebra is solvable by [13, Theorem 6.19].

2. Jordan operators. Recall that $T \in \mathbf{B}(\mathcal{X})$ is a Jordan operator if there exists a normal-equivalent operator $S \in \mathbf{B}(\mathcal{X})$ and a quasinilpotent operator $Q \in \mathbf{B}(\mathcal{X})$ such that $[S, Q]=0$ and $T=S+Q$. This last formula is called the Jordan decomposition of $T$. It is known that every Jordan operator is a completely regular generalized spectral operator (cf. $[4, \S 14$, Corollary 5]).

Lemma 2.1 ([4, $\S 14$, Theorem 7$])$. Let $T \in \mathbf{B}(\mathcal{X})$ be a Jordan operator with the Jordan decomposition $T=S+Q$. Moreover let $A, B \in \mathbf{B}(\mathcal{X})$ be hermitian-equivalent operators such that $S=A+i B$ and $[A, B]=0$. Then $A, B$ and $Q$ belong to the bicommutant of $T$. Moreover, $\mathcal{B}_{\mathrm{ad} T}(F)=\mathcal{B}_{\mathrm{ad} S}(F)$ for every closed subset $F$ of $\mathbb{C}$. 
The last statement of Lemma 2.1 is in the proof of $[4, \S 14$, Theorem 7]. Recall that if $\mathcal{L}$ is a Lie algebra, then $\mathcal{A}(\mathcal{L})$ denotes the associative algebra generated by $\mathcal{L}, \overline{\mathcal{A}(\mathcal{L})}$ the Banach algebra generated by $\mathcal{L}$, and $\operatorname{Rad}(\overline{\mathcal{A}(\mathcal{L})})$ the Jacobson radical of $\overline{\mathcal{A}(\mathcal{L})}$. A Banach algebra $\mathcal{B}$ is called $E n$ gel if ad $a: \mathcal{B} \rightarrow \mathcal{B}$ is quasinilpotent for every $a \in \mathcal{B}$. It is well known that if $\mathcal{B}$ is an Engel algebra, then $\mathcal{B} / \operatorname{Rad} \mathcal{B}$ is commutative (see $[13$, Proposition 5.21] or [3, Proposition]).

The following lemma can be found in [10].

Lemma 2.2. If $A$ is a Banach algebra and $A / \operatorname{Rad} A$ is commutative, then $Q_{A}=\operatorname{Rad}(A)$, where $Q_{A}$ is the set of all quasinilpotent elements in $A$.

The following lemma can be found in [14].

Lemma 2.3. If $\mathcal{L} \subset \mathbf{B}(\mathcal{X})$ is a nilpotent (or finite-dimensional solvable) Lie algebra, then $\overline{\mathcal{A}(\mathcal{L})} / \operatorname{Rad}(\overline{\mathcal{A}(\mathcal{L})})$ is commutative.

If $\mathcal{L}$ is an Engel Lie algebra, will $\overline{\mathcal{A}(\mathcal{L})}$ be an Engel algebra? This question was posed by Yu. V. Turovskiı̌ and V. S. Shulman in [16], and a partial answer can be found in [13].

Lemma 2.4 ([13, Theorem 5.22]). Let $\mathcal{L} \subset \mathcal{K}^{1}(\mathcal{X})$ be an Engel Lie algebra, where $\mathcal{K}^{1}(\mathcal{X})$ is the linear space generated by the compact operators and the identity operator on $\mathcal{X}$. Then $\overline{\mathcal{A}(\mathcal{L})}$ is an Engel algebra.

Now we begin the study of Lie algebras generated by Jordan operators.

First, note that if a Lie algebra generated by normal operators in a Hilbert space is finite-dimensional solvable, then it is commutative ([5, Theorem 2.1]). We will generalize this result to normal operators on Banach spaces. The following proposition is the pivotal step.

For two hermitian operators $N_{1}, N_{2}, i\left[N_{1}, N_{2}\right]$ is a hermitian operator. In fact, since

$\exp \left(\left[N_{1}, N_{2}\right]\right)$

$$
=\lim _{n \rightarrow \infty}\left(\exp \left(\frac{1}{n} N_{1}\right) \exp \left(\frac{1}{n} N_{2}\right) \exp \left(-\frac{1}{n} N_{1}\right) \exp \left(-\frac{1}{n} N_{2}\right)\right)^{n^{2}},
$$

we have $\left\|\exp \left(t\left[N_{1}, N_{2}\right]\right)\right\|=\left\|\exp \left(-t\left[i N_{1}, i N_{2}\right]\right)\right\| \leq 1$ for every $t \in \mathbb{R}$. But $1=\left\|\exp \left(t\left[N_{1}, N_{2}\right]\right) \exp \left(-t\left[N_{1}, N_{2}\right]\right)\right\| \leq 1$, so $\left\|\exp \left(t\left[N_{1}, N_{2}\right]\right)\right\|=1$ for every $t \in \mathbb{R}$. Hence $i\left[N_{1}, N_{2}\right]$ is hermitian (cf. [4, $\S 14$, Remark 5]).

Proposition 2.1. If $N_{1}, N_{2} \in \mathbf{B}(\mathcal{X})$ are hermitian, and $\varepsilon\left(N_{1}, N_{2}\right)$ is an ad-compact E-solvable Lie algebra, then $N_{1} N_{2}=N_{2} N_{1}$.

Proof. Let $\mathcal{L}=\varepsilon\left(N_{1}, N_{2}\right)$. Because $\mathcal{L}$ is ad-compact E-solvable, $[\mathcal{L}, \mathcal{L}]$ is an Engel Lie algebra by [13, Theorem 6.15]. So $\operatorname{ad}\left(i\left[N_{1}, N_{2}\right]\right)$ is quasinilpotent on $[\mathcal{L}, \mathcal{L}]$. Since $i\left[N_{1}, N_{2}\right]$ is hermitian, $\operatorname{ad}\left(i\left[N_{1}, N_{2}\right]\right)$ is normal. By 
Lemma $1.3,[\mathcal{L}, \mathcal{L}] \subset \mathcal{B}_{\text {ad }\left(i\left[N_{1}, N_{2}\right]\right)}(\{0\})$, so $\operatorname{ad}\left(\left[N_{1}, N_{2}\right]\right)=0$ on $[\mathcal{L}, \mathcal{L}]$ by Lemma 1.2. Because $\left[\left[N_{1}, N_{2}\right], N_{1}\right] \in[\mathcal{L}, \mathcal{L}]$, we have

$$
\operatorname{ad}\left(\left[N_{1}, N_{2}\right]\right)\left(\left[\left[N_{1}, N_{2}\right], N_{1}\right]\right)=\operatorname{ad}^{2}\left(\left[N_{1}, N_{2}\right]\right)\left(N_{1}\right)=0 .
$$

Now $N_{1} \in \mathcal{B}_{\mathrm{ad}\left(\left[N_{1}, N_{2}\right]\right)}(\{0\})$, by Lemma 1.3. So $\operatorname{ad}\left(\left[N_{1}, N_{2}\right]\right)\left(N_{1}\right)=0$ by Lemma 1.2. That is, $\operatorname{ad}^{2}\left(N_{1}\right)\left(N_{2}\right)=0$. Again $N_{2} \in \mathcal{B}_{\text {ad } N_{1}}(\{0\})$, so that $\left[N_{1}, N_{2}\right]=0$.

Corollary 2.1. If $N_{1}, N_{2} \in \mathbf{B}(\mathcal{X})$ are hermitian, and $\varepsilon\left(N_{1}, N_{2}\right)$ is a finite-dimensional solvable Lie algebra, then $N_{1} N_{2}=N_{2} N_{1}$.

Proof. This is evident by Proposition 2.1 and [13, Theorem 6.19].

Theorem 2.1. If $N_{1}, N_{2} \in \mathbf{B}(\mathcal{X})$ are normal, and $\varepsilon\left(N_{1}, N_{2}\right)$ is finitedimensional solvable, then it is commutative, that is, $\left[N_{1}, N_{2}\right]=0$.

Proof. With Lemma 1.4 replacing the Fuglede-Putnam theorem in Hilbert space, the proof is similar to the proof of Theorem 2.1 in [15], so we omit it. See [5] for the details.

Lemma 2.5. For Jordan operators $J_{i}$ with the Jordan decomposition $J_{i}=$ $N_{i}+Q_{i}, i=1,2$, if $\varepsilon\left(J_{1}, J_{2}\right)$ is an Engel Lie algebra, then $\left[N_{1}, N_{2}\right]=0$, $\left[N_{1}, Q_{2}\right]=0$ and $\left[N_{2}, Q_{1}\right]=0$.

Proof. Since $\varepsilon\left(J_{1}, J_{2}\right)$ is an Engel Lie algebra, ad $J_{1}$ is a quasinilpotent operator on $\varepsilon\left(J_{1}, J_{2}\right)$. So $J_{2} \in \mathcal{B}_{\text {ad } J_{1}}(\{0\})$. By Lemma $2.1, J_{2} \in \mathcal{B}_{\text {ad } N_{1}}(\{0\})$, and by Lemma $1.2,\left[N_{1}, J_{2}\right]=0$. Note that $J_{2}$ is a Jordan operator, so $\left[N_{1}, N_{2}\right]=0$ and $\left[N_{1}, Q_{2}\right]=0$ by Lemma 2.1. Similarly, $\left[N_{2}, Q_{1}\right]=0$.

It is known that if $G$ is a finite-dimensional solvable Lie algebra in $\mathbf{B}(\mathcal{X})$, then the set of Jordan operators in $G$ is an ideal of $G$ (cf. [4, $\S 28$, Theorem 3]). Now we will give another property of Jordan operators in finite-dimensional solvable Lie algebras.

Proposition 2.2. For Jordan operators $J_{i}$ with the Jordan decomposition $J_{i}=N_{i}+Q_{i}, i=1,2$, suppose that $N_{1}, N_{2}$ are normal operators, and the Lie algebra $\varepsilon\left(J_{1}, J_{2}\right)$ is finite-dimensional solvable. Then $\left[N_{1}, N_{2}\right]=0$ and $Q_{1}, Q_{2} \in \operatorname{Rad}\left(\overline{\mathcal{A}\left(\left\{N_{1}, N_{2}, Q_{1}, Q_{2}\right\}\right)}\right)$.

Proof. Let $\mathcal{L}=\varepsilon\left(J_{1}, J_{2}\right)$ and $\left\{\lambda_{1}, \ldots, \lambda_{n}\right\}=\sigma\left(\operatorname{ad}_{\mathcal{L}} J_{1}\right)$, so $\operatorname{ad}_{\mathcal{L}} J_{1}$ has finite rank and $\mathcal{L}=\mathcal{B}_{\mathrm{ad}_{\mathcal{L}} J_{1}}\left(\lambda_{1}\right) \oplus \cdots \oplus \mathcal{B}_{\mathrm{ad}_{\mathcal{L}} J_{1}}\left(\lambda_{n}\right)$. By Lemmas 2.1 and 1.2, we have

$$
\mathcal{L}=\operatorname{Ker}\left(\operatorname{ad}_{\mathcal{L}} N_{1}-\lambda_{1}\right) \oplus \cdots \oplus \operatorname{Ker}\left(\operatorname{ad}_{\mathcal{L}} N_{1}-\lambda_{n}\right) .
$$

It is easy to see that $\operatorname{span}\left(N_{1}, \mathcal{L}\right)$ is a Lie algebra and

$$
\left[\operatorname{span}\left(N_{1}, \mathcal{L}\right), \operatorname{span}\left(N_{1}, \mathcal{L}\right)\right] \subset[\mathcal{L}, \mathcal{L}] .
$$

Since $\mathcal{L}$ is finite-dimensional solvable, it follows that $[\mathcal{L}, \mathcal{L}]$ is nilpotent, hence also $\left[\operatorname{span}\left(N_{1}, \mathcal{L}\right), \operatorname{span}\left(N_{1}, \mathcal{L}\right)\right]$ is nilpotent, and therefore $\operatorname{span}\left(N_{1}, \mathcal{L}\right)$ is 
finite-dimensional solvable. Similarly, $\operatorname{span}\left(N_{1}, N_{2}, \mathcal{L}\right)$ is finite-dimensional solvable. So also is $\varepsilon\left(N_{1}, N_{2}\right)$. Therefore, $\left[N_{1}, N_{2}\right]=0$ by Theorem 2.1. Since $Q_{1}, Q_{2} \in \operatorname{span}\left(N_{1}, N_{2}, \mathcal{L}\right)$, Lemmas 2.2 and 2.3 show that $Q_{1}, Q_{2} \in$ $\operatorname{Rad}\left(\overline{\mathcal{A}\left(\left\{N_{1}, N_{2}, Q_{1}, Q_{2}\right\}\right)}\right)$.

Recall that for $T \in \mathcal{L}, \operatorname{ad} T: \mathcal{L} \rightarrow \mathcal{L}$ is quasinilpotent if and only if $\operatorname{ad} T: \overline{\mathcal{L}} \rightarrow \overline{\mathcal{L}}$ is quasinilpotent if and only if $\left\|(\operatorname{ad} T)^{n}(S)\right\|^{1 / n} \rightarrow 0$ as $n \rightarrow \infty$ for any $S \in \overline{\mathcal{L}}$. A Volterra ideal is an ideal consisting of Volterra operators.

Recall that a Jordan operator $J$ on a Hilbert space $\mathscr{H}$ has the Jordan decomposition $J=N+Q$, where $N \in \mathbf{B}(\mathscr{H})$ is normal, $Q \in \mathbf{B}(\mathscr{H})$ is quasinilpotent and $[N, Q]=0$.

TheOREM 2.2. For Jordan operators $J_{i}$ on a Hilbert space with the Jordan decomposition $J_{i}=N_{i}+Q_{i}, i=1,2$, suppose that $\varepsilon\left(J_{1}, J_{2}\right)$ is an Engel Lie algebra, and $Q_{1}, Q_{2}$ are Volterra operators. Then $\overline{\mathcal{A}\left(\left\{N_{1}, N_{2}, Q_{1}, Q_{2}\right\}\right)}$ is an Engel algebra, as also is $\overline{\mathcal{A}\left(\varepsilon\left(J_{1}, J_{2}\right)\right)}$.

Proof. By Lemma 2.5, $\left[N_{1}, N_{2}\right]=0,\left[N_{1}, Q_{2}\right]=0$ and $\left[N_{2}, Q_{1}\right]=0$. Let $\mathcal{L}=\operatorname{span}\left(N_{1}, N_{2}, \varepsilon\left(J_{1}, J_{2}\right)\right)$.

Claim 1. $\mathcal{L}$ is an Engel Lie algebra.

Note that $\left[N_{1}, J_{1}\right]=0=\left[N_{1}, J_{2}\right]$, so ad $N_{1}=0$ on $\mathcal{L}$. Similarly, ad $N_{2}=0$ on $\mathcal{L}$. For every $T$ in $\varepsilon\left(J_{1}, J_{2}\right)$, since $\varepsilon\left(J_{1}, J_{2}\right)$ is an Engel Lie algebra, $\lim _{n \rightarrow \infty}\left\|(\operatorname{ad} T)^{n}(S)\right\|^{1 / n}=0$ for every $S \in \overline{\varepsilon\left(J_{1}, J_{2}\right)}$. As

$$
\overline{\mathcal{L}}=\operatorname{span}\left(N_{1}, N_{2}, \overline{\varepsilon\left(J_{1}, J_{2}\right)}\right),
$$

it follows that $\lim _{n \rightarrow \infty}\left\|(\operatorname{ad} T)^{n}(S)\right\|^{1 / n}=0$ for every $S \in \overline{\mathcal{L}}$. That is, $\operatorname{ad} T$ is a quasinilpotent operator on $\mathcal{L}$. For every $T^{\prime} \in \mathcal{L}$ we have $T^{\prime}=\lambda_{1} N_{1}+$ $\lambda_{2} N_{2}+T$, where $T \in \varepsilon\left(J_{1}, J_{2}\right)$. So $\operatorname{ad} T^{\prime}=\operatorname{ad} T$ on $\mathcal{L}$ is a quasinilpotent operator. That is, $\mathcal{L}$ is an Engel Lie algebra.

Claim 2. $\overline{\mathcal{A}\left(\varepsilon\left(Q_{1}, Q_{2}\right)\right)}$ consists of quasinilpotent operators.

$\varepsilon\left(Q_{1}, Q_{2}\right)$ is an Engel Lie algebra since it is contained in $\mathcal{L}$. As $Q_{1}, Q_{2}$ are compact operators, so is every operator in $\varepsilon\left(Q_{1}, Q_{2}\right)$. So by [13, Theorem 5.22], $\overline{\mathcal{A}\left(\varepsilon\left(Q_{1}, Q_{2}\right)\right)}$ is an Engel algebra. Hence $\overline{\mathcal{A}\left(\varepsilon\left(Q_{1}, Q_{2}\right)\right)}$ consists of quasinilpotent operators by Lemmas 2.2 and 2.3.

Let $\mathcal{A}=\mathcal{A}\left(N_{1}, N_{2}, Q_{1}, Q_{2}\right)$.

Claim 3. $I:=\overline{\operatorname{span}\left(p\left(N_{1}, N_{2}\right) q\left(Q_{1}, Q_{2}\right)\right)}$ is a Volterra ideal of $\overline{\mathcal{A}}$, where $p\left(x_{1}, x_{2}\right)$ and $q\left(x_{1}, x_{2}\right)$ run through polynomials such that $q(0,0)=0$.

First, we show that $I$ is an ideal of $\overline{\mathcal{A}}$. Note that $\left[N_{1}, N_{2}\right]=0=\left[N_{i}, Q_{j}\right]$ for every $i, j=1,2$. Every $S \in \mathcal{A}$ has the form $\sum_{i=1}^{n} p_{i}\left(N_{1}, N_{2}\right) q_{i}\left(Q_{1}, Q_{2}\right)$, where $p_{i}\left(x_{1}, x_{2}\right), q_{i}\left(x_{1}, x_{2}\right)$ are polynomials and $p_{i}(0,0) q_{i}(0,0)=0$. So it is 
easy to see that $S I, I S \subset I$. Note that $I$ is closed, so $\overline{\mathcal{A}} I, I \overline{\mathcal{A}} \subset I$. That is, $I$ is an ideal of $\overline{\mathcal{A}}$.

Next we show that $I$ consists of Volterra operators. Note that an operator $p\left(N_{1}, N_{2}\right) q\left(Q_{1}, Q_{2}\right) \in I$ is compact and quasinilpotent, since $q\left(Q_{1}, Q_{2}\right)$ is compact and quasinilpotent, and $\left[p\left(N_{1}, N_{2}\right), q\left(Q_{1}, Q_{2}\right)\right]=0$. For any $n$ operators $p_{i}\left(N_{1}, N_{2}\right) q_{i}\left(Q_{1}, Q_{2}\right) \in I$, where $q_{i}(0,0)=0, i=1, \ldots, n$, since $\overline{\mathcal{A}\left(Q_{1}, Q_{2}\right)}$ consists of quasinilpotent operators and $\left[\mathcal{A}\left(N_{1}, N_{2}\right), \mathcal{A}\left(Q_{1}, Q_{2}\right)\right]$ $=\{0\}$, the semigroup generated by $p_{i}\left(N_{1}, N_{2}\right) q_{i}\left(Q_{1}, Q_{2}\right)$ consists of Volterra operators. Hence so does the algebra generated by $p_{i}\left(N_{1}, N_{2}\right) q_{i}\left(Q_{1}, Q_{2}\right)$, $i=1, \ldots, n\left(\left[15\right.\right.$, Theorem 4]). That is, $\operatorname{span}\left(p\left(N_{1}, N_{2}\right) q\left(Q_{1}, Q_{2}\right)\right)$ consists of Volterra operators, where $p\left(x_{1}, x_{2}\right), q\left(x_{1}, x_{2}\right)$ run through polynomials such that $q(0,0)=0$.

Finally, since the limit of Volterra operators is a Volterra operator, $I$ consists of Volterra operators.

By the definition of Jacobson radical, $I \subset \operatorname{Rad}(\overline{\mathcal{A}})$, so $Q_{1}, Q_{2} \in \operatorname{Rad}(\overline{\mathcal{A}})$.

Claim 4. For every $S \in \mathcal{A}$, ad $S: \overline{\mathcal{A}} \rightarrow \overline{\mathcal{A}}$ is quasinilpotent.

Since $S=\sum_{i=1}^{n} p_{i}\left(N_{1}, N_{2}\right) q_{i}\left(Q_{1}, Q_{2}\right)$, where $p_{i}\left(x_{1}, x_{2}\right), q_{i}\left(x_{1}, x_{2}\right)$ are polynomials and $p_{i}(0,0) q_{i}(0,0)=0$, we have $S=S_{1}+S_{2}$, where $S_{1} \in$ $\mathcal{A}\left(N_{1}, N_{2}\right)$ and $S_{2} \in I$. Note that ad $N_{i}(\overline{\mathcal{A}})=\{0\}$, and $I$ consists of Volterra operators by Claim 3, so ad $S=\operatorname{ad} S_{2}$ is a quasinilpotent operator on $\bar{A}$, by Rosenblum's theorem.

Claim 5. $\overline{\mathcal{A}} \subset C^{*}\left(N_{1}, N_{2}\right)+\mathcal{K}(\mathscr{H})$, where $C^{*}\left(N_{1}, N_{2}\right)$ is the $C^{*}$-algebra generated by $N_{1}, N_{2}$, and $\mathcal{K}(\mathscr{H})$ is the set of compact operators on $\mathscr{H}$.

It is well known that the sum of a $C^{*}$-algebra and $\mathcal{K}(\mathscr{H})$ is closed in $\mathbf{B}(\mathscr{H})$ [7]. So $C^{*}\left(N_{1}, N_{2}\right)+\mathcal{K}(\mathscr{H})$ is closed. Note that $\mathcal{A} \subset \mathcal{A}\left(N_{1}, N_{2}\right)+I$, and $I$ consists of Volterra operators, so $\mathcal{A} \subset C^{*}\left(N_{1}, N_{2}\right)+\mathcal{K}(\mathscr{H})$. As $C^{*}\left(N_{1}, N_{2}\right)+\mathcal{K}(\mathscr{H})$ is closed, it follows that $\overline{\mathcal{A}} \subset C^{*}\left(N_{1}, N_{2}\right)+\mathcal{K}(\mathscr{H})$.

Now, for every $S^{\prime} \in \overline{\mathcal{A}}$, by Claim 5 , there exist $N^{\prime} \in C^{*}\left(N_{1}, N_{2}\right)$ and $Q^{\prime} \in \mathcal{K}(\mathscr{H})$, such that $S^{\prime}=N^{\prime}+Q^{\prime}$. Note that $\left[N_{i}, \overline{\mathcal{A}}\right]=\{0\}$ and $N_{i}$ is normal, so $\left[N_{i}^{*}, \overline{\mathcal{A}}\right]=\{0\}, i=1,2$. Therefore, ad $S^{\prime}=\operatorname{ad} N^{\prime}+\operatorname{ad} Q^{\prime}=\operatorname{ad} Q^{\prime}$ on $\overline{\mathcal{A}}$. But $Q^{\prime}$ is a compact operator, so ad $Q^{\prime}=\operatorname{ad} S^{\prime}$ has countable spectrum on $\overline{\mathcal{A}}$. Since $S^{\prime} \in \overline{\mathcal{A}}$, there is a sequence $\left\{S_{n}\right\} \subset \mathcal{A}$ such that $\lim _{n \rightarrow \infty} S_{n}=S^{\prime}$. Hence, $\lim _{n \rightarrow \infty}$ ad $S_{n}=$ ad $S^{\prime}$. By Claim 4 , ad $S_{n}$ is quasinilpotent on $\overline{\mathcal{A}}$; as ad $S^{\prime}$ has countable spectrum on $\overline{\mathcal{A}}$, it is quasinilpotent on $\overline{\mathcal{A}}$ by Newburgh's result [11]. That is, $\overline{\mathcal{A}}$ is an Engel algebra.

The last statement of the theorem is clear because $\overline{\mathcal{A}\left(\varepsilon\left(J_{1}, J_{2}\right)\right)}$ is a subalgebra of $\overline{\mathcal{A}}$.

Now we turn to Jordan operators on Banach spaces. We adopt the notation of the proof of Theorem 2.2. 
COROLlary 2.2. For Jordan operators $J_{i}$ with the Jordan decomposition $J_{i}=N_{i}+Q_{i}, i=1,2$, suppose that $\varepsilon\left(J_{1}, J_{2}\right)$ is an Engel Lie algebra, and $Q_{1}, Q_{2}$ are Volterra operators. Then $\overline{\mathcal{A}} / \operatorname{Rad}(\overline{\mathcal{A}})$ is commutative.

Proof. Note that Claim 3 in the proof of Theorem 2.2 holds for Jordan operators on Banach spaces, and $\mathcal{A} \subset \mathcal{A}\left(N_{1}, N_{2}\right)+I$ with $\left[N_{i}, \overline{\mathcal{A}}\right]=\{0\}$, $i=1,2$. So $[\mathcal{A}, \mathcal{A}] \subset I \subset \operatorname{Rad}(\overline{\mathcal{A}})$ by Claim 3. As $\operatorname{Rad}(\overline{\mathcal{A}})$ is closed, also $[\overline{\mathcal{A}}, \overline{\mathcal{A}}] \subset \operatorname{Rad}(\overline{\mathcal{A}})$

COROLlary 2.3. For Jordan operators $J_{i}$ with the Jordan decomposition $J_{i}=N_{i}+Q_{i}, i=1,2$, suppose that $\varepsilon\left(J_{1}, J_{2}\right)$ is an Engel Lie algebra, and $Q_{1}, Q_{2}$ are compact quasinilpotent operators. Then every operator in $\varepsilon\left(J_{1}, J_{2}\right)$ is a Jordan operator.

Proof. It is easy to see that every scalar multiple of a Jordan operator is a Jordan operator. By Corollary 2.2, $Q_{1}+Q_{2}$ and the commutator of any two operators in $\varepsilon\left(J_{1}, J_{2}\right)$ are quasinilpotent operators. It remains to prove that $J_{1}+J_{2}$ is a Jordan operator. But by Lemma $2.5,\left[N_{1}, N_{2}\right]=0$, so by $[4, \S 14$, Corollary 6], $N_{1}+N_{2}$ is a normal operator. Note that $\left[N_{1}+N_{2}, Q_{1}+Q_{2}\right]=0$, and $Q_{1}+Q_{2}$ is quasinilpotent, so $J_{1}+J_{2}$ is a Jordan operator.

Corollary 2.4. For Jordan operators $J_{i}$ with the Jordan decomposition $J_{i}=N_{i}+Q_{i}, i=1,2$, suppose that $\varepsilon\left(J_{1}, J_{2}\right)$ is an Engel Lie algebra, and $Q_{1}, Q_{2}$ are Volterra operators. Then $\mathcal{A}$ is reduced.

Proof. By Claim 3 in the proof of Theorem 2.2, $I$ is a nonzero Volterra ideal of $\overline{\mathcal{A}}$. So $\overline{\mathcal{A}}$ has a nontrivial hyperinvariant subspace by Shulman's result $[14]$.

REMARK 2.1. Because there is a nil algebra of operators on a Hilbert space with semisimple norm closure, there is a Lie algebra $\mathcal{L}$ generated by Jordan operators which is an Engel Lie algebra, but $\overline{\mathcal{A}(\mathcal{L})}$ is not an Engel algebra (see [9]).

Acknowledgments. The authors would like to thank A. Dosiev and D. Beltiţă for valuable suggestions, and Yu. V. Turovskiı̆ and V. S. Shulman for sending their useful papers. We also thank the referee for useful comments.

\section{References}

[1] E. Albrecht, On some classis of generalized spectral operaotrs, Arch. Math. (Basel) 30 (1978), 297-303.

[2] E. Albrecht and P. G. Spain, When products of selfadjoints are normal, Proc. Amer. Math. Soc. 128 (2000), 2509-2511.

[3] B. Aupetit and M. Mathieu, The continuity of Lie homomorphisms, Studia Math. 138 (2000), 193-199. 
[4] D. Beltiţă and M. Şabac, Lie Algebras of Bounded Operators, Birkhäuser, 2001.

[5] P. Cao and S. L. Sun, Finite dimensional solvable Lie algebras generated by normal operators are commutative, J. Math. Anal. Appl. 337 (2008), 928-931.

[6] I. Colojoară and C. Foiaş, Theory of Generalized Spectral Operators, Gordon and Breach, 1968.

[7] J. Dixmier, Les $C^{*}$-Algèbres et Leurs Représentations, Gauthier-Villars, Paris, 1964.

[8] H. R. Dowson, Spectral Theory of Linear Operators, Academic Press, 1978.

[9] D. Hadwin, E. Nordgren, M. Radjabalipour, H. Radjavi, and P. Rosenthal. A nil algebra of operators on Hilbert spaces with semisimple norm closure, Integral Equations Operator Theory 9 (1986), 729-743.

[10] A. Katavolos and C. Stamatopoulos, Commutators of quasinilpotents and invariant subspaces, Studia Math. 128 (1998), 159-169.

[11] J. Newburgh, The variation of spectra, Duke Math. J. 18 (1951), 165-176.

[12] V. S. Shulman, On invariant subspaces of Volterra operators, Funktsional. Anal. i Prilozhen. 18 (1984), no. 2, 84-85 (in Russian).

[13] V. S. Shulman and Yu. V. Turovskiǔ, Invariant subspaces of operator Lie algebras and Lie algebras with compact adjoint action, J. Funct. Anal. 223 (2005), 425-508.

[14] Yu. V. Turovskiı̌, Spectral properties of certain Lie subalgebras and the spectral radius of subsets of a Banach algebra, in: Spectral Theory of Operators and its Applications, F. G. Maksudov (ed.), vol. 6, Elm, Baku, 1985, 144-181 (in Russian).

[15] -, Volterra semigroups have invariant subspaces, J. Funct. Anal. 126 (1999), 313322.

[16] Yu. V. Turovskiı̌ and V. S. Shulman, Radicals in Banach algebras and some problems in the theory of radical Banach algebras, Funct. Anal. Appl. 35 (2001), 312-314.

Department of Mathematics

Beijing Institute of Technology

Beijing, China, 100081

E-mail: cpeng@bit.edu.cn
LMIB \& Department of Mathematics Beihang University Beijing, China, 100083

E-mail: shlsuncn@yahoo.com.cn

Received March 17, 2007

Revised version February 11, 2008 